\title{
The Dynamics and Synchronization of a Fractional-Order System with Complex Variables
}

\author{
Xiaoya Yang, Xiaojun Liu, Honggang Dang, and Wansheng He \\ School of Mathematics and Statistics, Tianshui Normal University, Tianshui 741001, China \\ Correspondence should be addressed to Xiaojun Liu; flybett3952@126.com
}

Received 15 May 2014; Accepted 10 July 2014; Published 21 July 2014

Academic Editor: Dumitru Baleanu

Copyright (C) 2014 Xiaoya Yang et al. This is an open access article distributed under the Creative Commons Attribution License, which permits unrestricted use, distribution, and reproduction in any medium, provided the original work is properly cited.

\begin{abstract}
A fractional-order system with complex variables is proposed. Firstly, the dynamics of the system including symmetry, equilibrium points, chaotic attractors, and bifurcations with variation of system parameters and derivative order are studied. The routes leading to chaos including the period-doubling and tangent bifurcations are obtained. Then, based on the stability theory of fractionalorder systems, the scheme of synchronization for the fractional-order complex system is presented. By designing appropriate controllers, the synchronization for the system is realized. Numerical simulations are carried out to demonstrate the effectiveness of the proposed scheme.
\end{abstract}

\section{Introduction}

Fractional calculus considered as the generalization of the integer-order calculus can be dated back to the 17th century. Recently, it has attracted many researchers' interest for the ability to describe practical problems. Moreover, rich dynamics such as chaos and bifurcation exist in many fractionalorder systems [1-3]. Meanwhile, compared with integerorder chaotic systems, fractional-order chaotic systems with more complexity dynamics characteristic and more system parameters can provide higher security for secure communication $[4,5]$.

Chaos synchronization has attracted increasing interests in recent years which was proposed in 1990 [6]. Synchronization of integer-order systems in real space and complex space has been studied extensively and several methods are extended to synchronize fractional-order complex systems [7-9]. It should be noted that fractional-order chaotic systems with complex variables (doubling the numbers of variables) can be used to increase the content of transmitting information signals and enhance their security further. Therefore, it is an interesting and meaningful topic for researchers to study the dynamics and synchronization for fractional-order complex nonlinear systems. Meanwhile, the chaos and synchronization for discrete fractional-order systems have been investigated in detail [10-13].
Motivated by the above discussion, a fractional-order system with complex variables is proposed. The dynamics of the system including symmetry, equilibrium points, chaotic attractors, periodic orbits, and bifurcations with variation of system parameters and derivative orders are studied. Furthermore, based on the stability theory of fractional-order systems, the scheme of synchronization for the fractionalorder complex system is presented, and numerical simulations are used to demonstrate the effectiveness and feasibility of the proposed scheme.

The paper is organized as follows. In Section 2, the definitions for fractional calculus and numerical algorithms are introduced. In Section 3, the fractional-order system with complex variables is proposed. And the dynamic behaviors of the system are investigated in detail. In Section 4, the synchronization of the system is investigated. Finally, we summarize the results in Section 5.

\section{Fractional Operators and Their Approximation}

2.1. Definition. There are at least six kinds of definitions for the fractional derivative. Three of which most frequently used ones are the Grunwald-Letnikov definition and the RiemannLiouville and the Caputo definitions [14]. 
The Grunwald-Letnikov definition (GL) derivative with fractional-order $q$ is expressed by

$$
{ }_{a}^{\mathrm{GL}} D_{t}^{q} f(t)=\lim _{h \rightarrow 0} \frac{1}{h^{q}} \sum_{j=0}^{[(t-a) / h]}(-1)^{j}\left(\begin{array}{c}
q \\
j
\end{array}\right) f(t-j h),
$$

where the symbol $[\cdot]$ means the integer part.

The Riemann-Liouville (RL) definition of fractional derivatives is described by

$$
\begin{aligned}
& { }_{a}^{\mathrm{RL}} D_{t}^{q} f(t) \\
& \quad=\frac{d^{n}}{d t^{n}} \frac{1}{\Gamma(n-q)} \int_{a}^{t} \frac{f(\tau)}{(t-\tau)^{q-n+1}} d \tau, \quad n-1<q<n,
\end{aligned}
$$

where $\Gamma(\cdot)$ is the gamma function.

The Caputo $(C)$ fractional derivative is defined as follows:

$$
\begin{array}{r}
{ }_{a}^{C} D_{t}^{q} f(t)=\frac{1}{\Gamma(n-q)} \int_{a}^{t}(t-\tau)^{n-q-1} f^{(n)}(\tau) d \tau, \\
n-1<q<n .
\end{array}
$$

It is well known that the initial conditions for the fractional differential equations with Caputo derivatives take on the same form as those for the integer-order ones, which is very suitable for practical problems [15]. Therefore, we will use the Caputo definition for the fractional derivatives in this paper.

2.2. Numerical Algorithms. Due to the nature of the nonlocality, the operator of fractional derivative is more complicated than the classical ones which cause the difficulty of obtaining the analytic solution. However, numerical solution can make us study this kind of derivative better.

Nowadays, there are two approximation methods which can frequently be used to numerical computation fractional differential equations. One is an improved version of AdamsBashforth-Moulton algorithm [16-18]. The other is a method, known as frequency domain approximation [19], based on numerical analysis of fractional-order systems in the frequency domain. And the reliability of the former has been widely approved. Therefore, we employ the improved predictor-corrector algorithm for fractional-order differential equations in this paper.

In order to get the approximate solution of a fractionalorder chaotic system by the improved predictor-corrector algorithm, the following equation is considered:

$$
\begin{gathered}
\frac{d^{q} x}{d t^{q}}=f(t, x), \quad 0 \leq t \leq T, \\
x^{(k)}(0)=x_{0}^{(k)}, \quad k=0,1, \ldots,\lceil q\rceil-1,
\end{gathered}
$$

where $\lceil q\rceil$ is just the value $q$ rounded up to the nearest integer and $x^{(k)}$ is the ordinary $k$ th derivative of $x$. Formula (4) is equivalent to the Volterra integral equation

$$
x(t)=\sum_{k=0}^{\lceil q\rceil-1} x_{0}^{(k)} \frac{t^{k}}{k !}+\frac{1}{\Gamma(q)} \int_{0}^{t}(t-\tau)^{q-1} f(\tau, x(\tau)) \mathrm{d} \tau .
$$

Now, for the sake of simplicity, we assume that we are working on a uniform grid $\left\{t_{n}=n h: n=0,1, \ldots, M\right\}$ with some integer $M$ and set $h=T / M$. Using the standard quadrature techniques for the integral in (5) and denoting $g(\tau)=f(\tau, x(\tau))$ the integral is replaced by the trapezoidal quadrature formula at point $t_{n+1}$ :

$$
\int_{0}^{t_{n+1}}\left(t_{n+1}-\tau\right)^{q-1} g(\tau) \mathrm{d} \tau \approx \int_{0}^{t_{n+1}}\left(t_{n+1}-\tau\right)^{q-1} \widetilde{g}_{n+1}(\tau) \mathrm{d} \tau,
$$

where $\widetilde{g}_{n+1}$ is the piecewise linear interpolant for $g$ with nodes and knots chosen at the $t_{j},(j=0,1, \ldots, n+1)$. After some elementary calculations, the right hand side of (6) gives

$$
\int_{0}^{t_{n+1}}\left(t_{n+1}-\tau\right)^{q-1} \tilde{g}_{n+1}(\tau) \mathrm{d} \tau=\frac{h^{q}}{q(q+1)} \sum_{j=0}^{n+1} \alpha_{j, n+1} g\left(t_{j}\right) .
$$

And if we use the product rectangle rule, the right hand side of (6) can be written as

$$
\int_{0}^{t_{n+1}}\left(t_{n+1}-\tau\right)^{q-1} \widetilde{g}_{n+1}(\tau) \mathrm{d} \tau=\sum_{j=0}^{n+1} \beta_{j, n+1} g\left(t_{j}\right),
$$

where

$$
\begin{aligned}
& \alpha_{j, n+1} \\
& = \begin{cases}n^{q+1}-(n-q)(n+1)^{q}, & j=0, \\
(n-j+2)^{q+1}+(n-j)^{q+1} & \\
-2(n-j+1)^{q+1}, & 1 \leq j \leq n, \\
1, & j=n+1,\end{cases} \\
& \beta_{j, n+1}=\frac{h^{q}}{q}\left((n-j+1)^{q}-(n-j)^{q}\right), \quad 1 \leq j \leq n .
\end{aligned}
$$

Then the predictor and corrector formulae for solving (5) are given, respectively, by

$$
\begin{aligned}
x_{h}^{P}\left(t_{n+1}\right)= & \sum_{k=0}^{\lceil q\rceil-1} x_{0}^{(k)} \frac{t_{n+1}^{k}}{k !}+\frac{1}{\Gamma(q)} \sum_{j=0}^{n} \beta_{j, n+1} f\left(t_{j}, x_{h}\left(t_{j}\right)\right), \\
x_{h}\left(t_{n+1}\right)= & \sum_{k=0}^{\lceil q\rceil-1} x_{0}^{(k)} \frac{t_{n+1}^{k}}{k !}+\frac{h^{q}}{\Gamma(q+2)} f\left(t_{n+1}, x_{h}^{P}\left(t_{n+1}\right)\right) \\
& +\frac{h^{q}}{\Gamma(q+2)} \sum_{j=0}^{n} \alpha_{j, n+1} f\left(t_{j}, x_{h}\left(t_{j}\right)\right) .
\end{aligned}
$$

The approximation accuracy of scheme (10)-(11) is $O\left(h^{\min \{2,1+q\}}\right)$.

\section{Dynamics of a Fractional-Order Complex System}

A three-dimensional chaotic system was proposed in [20], and the dynamical behaviors of the system were studied in 


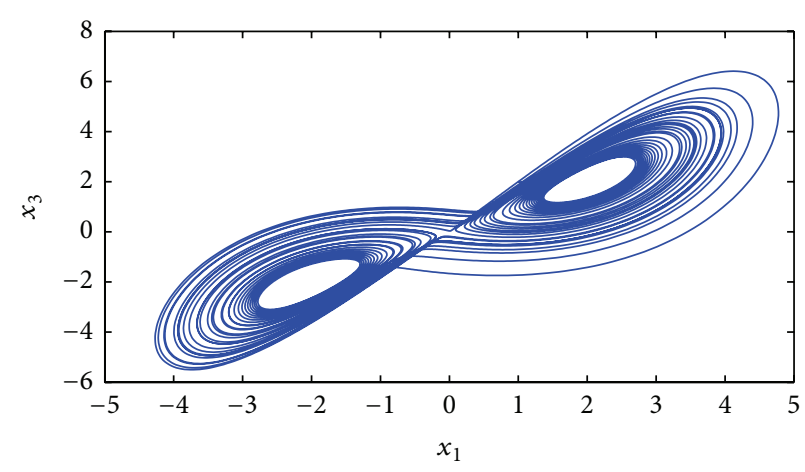

(a)

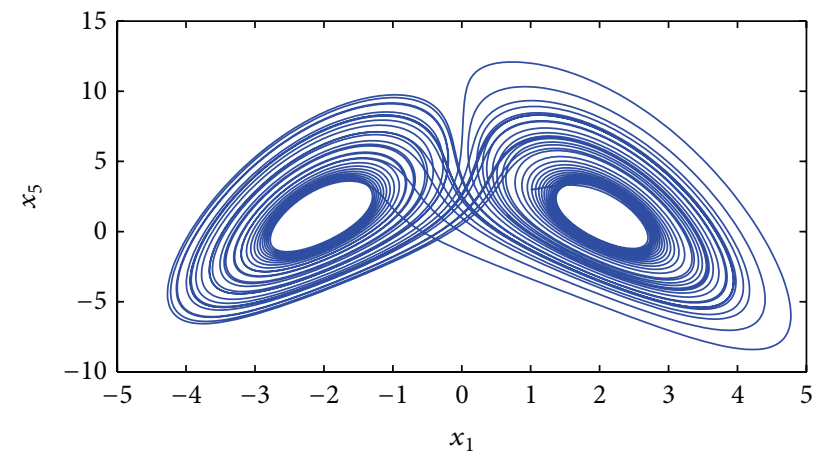

(b)

FIGURE 1: The chaotic attractors of the system (14) projected onto different phase plane (a) $x_{1}-x_{3}$ phase plane and (b) the chaotic attractor projected onto $x_{1}-x_{5}$ phase plane.

detail. The system can be described by the following differential equations:

$$
\begin{gathered}
\dot{y}_{1}=a\left(y_{2}-y_{1}\right), \\
\dot{y}_{2}=y_{1} y_{3}-y_{2}, \\
\dot{y}_{3}=b-y_{1} y_{2}-c y_{3},
\end{gathered}
$$

where $y=\left(y_{1}, y_{2}, y_{3}\right)^{T}$ is the state variable vector of the system and $a, b, c$ are parameters. The system possesses an attractor when the parameters are as follows: $a=5, b=16$, $c=1$.

In this paper, we suppose the state variables of system (12) are defined in the complex field, and the derivate orders are fractional. Then the corresponding fractional-order system is defined as

$$
\begin{gathered}
D^{q} y_{1}=a\left(y_{2}-y_{1}\right), \\
D^{q} y_{2}=y_{1} y_{3}-y_{2}, \\
D^{q} y_{3}=b-\frac{1}{2}\left(\bar{y}_{1} y_{2}+y_{1} \bar{y}_{2}\right)-c y_{3},
\end{gathered}
$$

where $q$ is the derivative order and $y=\left(y_{1}, y_{2}, y_{3}\right)^{T}$ is the vector of state variables. $y_{1}=x_{1}+i x_{2}, y_{2}=x_{3}+i x_{4}$ are complex variables, $y_{3}=x_{5}$ is real variable, and $i=\sqrt{-1}$. Then the complex variables are separated into the real and imaginary parts, respectively. Due to the linearity of the Caputo differential operator, the system (13) can be represented as

$$
\begin{aligned}
& D^{q} x_{1}=a\left(x_{3}-x_{1}\right), \\
& D^{q} x_{2}=a\left(x_{4}-x_{2}\right), \\
& D^{q} x_{3}=x_{1} x_{5}-x_{3}, \\
& D^{q} x_{4}=x_{2} x_{5}-x_{4}, \\
& D^{q} x_{5}=b-\left(x_{1} x_{3}+x_{2} x_{4}\right)-c x_{5} .
\end{aligned}
$$

The system (14) is more convenient than system (13) for analysis and numerical simulation. When the system parameters are taken as $a=5, b=16, c=1$ and order of derivative $q=0.99$, the maximum Lyapunov exponent by numerical computation is $\lambda_{1}=0.071$, which means that the system (14) is chaotic. The chaotic attractors in different phase space projections are shown in Figure 1.

3.1. Symmetry and Equilibrium Points. Firstly, the system (14) is symmetric because the transformation

$$
S:\left(x_{1}, x_{2}, x_{3}, x_{4}, x_{5}\right) \longrightarrow\left(-x_{1},-x_{2},-x_{3},-x_{4}, x_{5}\right),
$$

which permits the system (14), is invariant for all values of parameters with the transformation. And the transformation $S$ also implies that the system is symmetric about $x_{5}$-axis; that is, $S(\psi)$ is one solution of the system (14) if $\psi$ is one of it.

Now, we turn to investigate the equilibrium points of the system (14), which can be obtained by solving the equations $D^{q} x_{j}=0,(j=1, \ldots, 5)$. Therefore, the equilibriums are

$$
E_{1}(0,0,0,0,0)
$$

$$
E_{\theta}(r \cos \theta, r \sin \theta, r \cos \theta, r \sin \theta, 1) \text {, }
$$

where $r=\sqrt{b-c}$ and $\theta \in[0,2 \pi]$. The equilibrium $E_{\theta}$ exists when the condition $b-c>0$ is satisfied.

3.2. Bifurcations. Firstly, the order of derivative is taken as $q=0.99$ and fix the parameters $b=16$ and $c=-0.2$. The bifurcation of system (14) as the parameter $a$ is varied is depicted in Figure 2. From the bifurcation diagram, it is clear that with the decrease of $a$ from 7.5 there is one limit cycle for a long parameter region until period-doubling bifurcation occurs when $a=6.45$. For the system, the route leading to chaos is a series of period-doubling bifurcations. The perioddoubling bifurcations can be observed when $a$ belongs to the intervals $[1,1.5]$ and $[4,4.5]$. Phase portraits are shown in Figure 3, from which we can see that the system exists period1 , period-2 for different values of the parameter $a$.

Secondly, the dynamics of system (14) varying the parameter $b$ will be investigated when $a=5, c=1$, and $q=0.99$. The bifurcation of the system (14) when the parameter $b$ is 


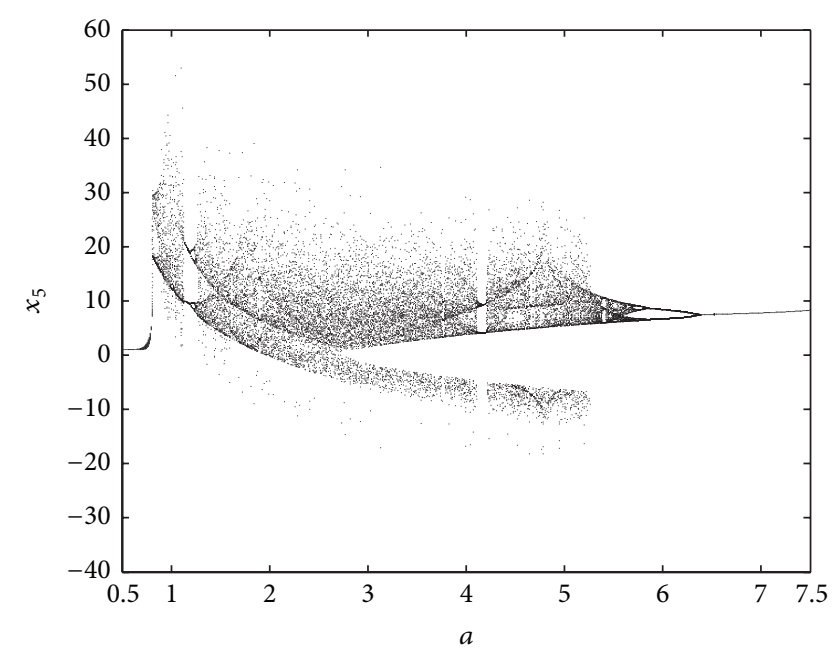

FIGURE 2: The bifurcation diagram of the system (14) with parameter $a \in[0.5,7.5]$.

varied from 10 to 125 is obtained by numerical computation, which is depicted in Figure 4. It is clear that with the decrease of the parameter $b$ the route out of chaos for the system is through tangent bifurcation when $b=110.5$. Then two limit cycles coexist until period-doubling bifurcation occurs when $b=105$. By a series of period-doubling bifurcations, the system enters into chaos again.

Thirdly, the dynamic behaviors of the system as the parameter $c$ varies will be studied when other system parameters are taken as $a=5, b=16$ and the derivative order $q=0.99$. The bifurcation diagram of the system (14) with parameter $c$ is depicted in Figure 5. The tangent bifurcation occurs when $c=0.05$. And the period-doubling bifurcations can be seen with $c \in[0.04,0.09]$.

It is well known that the derivative order is one of important parameters in dynamics analysis for fractionalorder systems. Therefore, the bifurcation diagram versus the derivative order with parameters $a=5, b=16$, and $c=-0.2$ is studied in the following.

The bifurcation diagram versus the order $q$ varying on the closed interval $[0.9,1]$ for the system (14) is depicted in Figure 6(a). The evolution of the period-doubling scenario and tangent bifurcation can be observed easily which is shown in the figure. In order to see clearly, the local of Figure 6(a) is zoomed in, which is shown in Figure 6(b). Meanwhile, to verify the chaotic behaviors for the system when $q \in[0.9,1]$, the corresponding largest Lyapunov exponent (LLE for short) diagram with the step size of 0.1 by the algorithm of small data sets $[21,22]$ is shown in Figure 6(c).

\section{Synchronization}

In this section, the synchronization for the fractional-order complex system (14) will be studied.

4.1. The Scheme of Synchronization. The synchronization scheme for fractional-order complex systems will be introduced. The drive and response systems are given, respectively, as follows:

$$
\begin{gathered}
D^{\alpha} y=f(y), \\
D^{\beta} Z=g(z)+u(y, z),
\end{gathered}
$$

where $\alpha \in(0,1)$ and $\beta \in(0,1)$ are the derivative orders of systems (17) and (18), respectively. $y=\left(y_{1}, y_{2}, \ldots y_{n}\right)^{T} \in R^{n}$ and $z=\left(z_{1}, z_{2}, \ldots z_{n}\right)^{T} \in R^{n}$ are state complex vectors of drive and response systems, and the complex variables can be defined as $y_{j}=x_{2 j-1}+i x_{2 j}$ and $z_{j}=x_{2 j-1}^{\prime}+i x_{2 j}^{\prime},(j=$ $1,2, \ldots, n)$. Nonlinear vector functions $f$ and $g$ are continuous. $u(y, z): R^{n} \times R^{n} \rightarrow R^{n}$ is a synchronization controller which will be designed later.

The error vector is defined as $e=z-y$, and the synchronization of drive and response systems is achieved if the following condition is satisfied:

$$
\lim _{t \rightarrow+\infty}\|e\|=\lim _{t \rightarrow+\infty}\|z-y\|=0
$$

where $\|\cdot\|$ is the Euclidean norm. For convenience, the error vector is rewritten as $e=e^{\text {real }}+i e^{\text {image }}, e^{\text {real }}=z^{\text {real }}-y^{\text {real }}$, and $e^{\text {image }}=z^{\text {image }}-y^{\text {image }}$.

Then, for the response system (18), we need to define a compensation controller

$$
\theta(y)=D^{\beta}(y)-g(y),
$$

and a synchronization controller

$$
u(y, z)=\theta(y)+\tau(y, z),
$$

where $\tau(y, z): R^{n} \times R^{n} \rightarrow R^{n}$ is a vector function that will be designed later for the realization of synchronization.

Substituting the controllers (20) and (21) into the response system (18), we can get the error dynamical system as follows:

$$
D^{\beta} e=g(z)-g(y)+\tau(y, z)=A(y, z) e+\tau(y, z) .
$$

For simplicity, $\tau(y, z)$ is chosen as $B(y, z) e$, and system (22) is rewritten as

$$
D^{\beta} e=A(y, z) e+B(y, z) e=[A(y, z)+B(y, z)] e ;
$$

then, the system (23) asymptotically converges to zero when error vector tends to zero for $t \rightarrow+\infty$.

Theorem 1. Given a fractional-order system (17), by designing a control vector $\tau(y, z)=B(y, z)$ e such that the synchronization between (17) and (18) can be realized, if the following condition is satisfied

$$
P[A(y, z)+B(y, z)]+[A(y, z)+B(y, z)]^{H} \mathbf{P}=-\mathbf{Q},
$$

where $\mathbf{P}$ and $\mathbf{Q}$ are real symmetric positive definite matrices, $B(y, z) \in R^{n \times n}$, and $H$ stands for conjugate transpose of a matrix.

Proof. For the error dynamical system (23), assume that $\lambda$ is one of the eigenvalues of matrix $[A(y, z)+B(y, z)]$, and the corresponding nonzero eigenvector is $\xi$; that is,

$$
[A(y, z)+B(y, z)] \xi=\lambda \xi \text {. }
$$




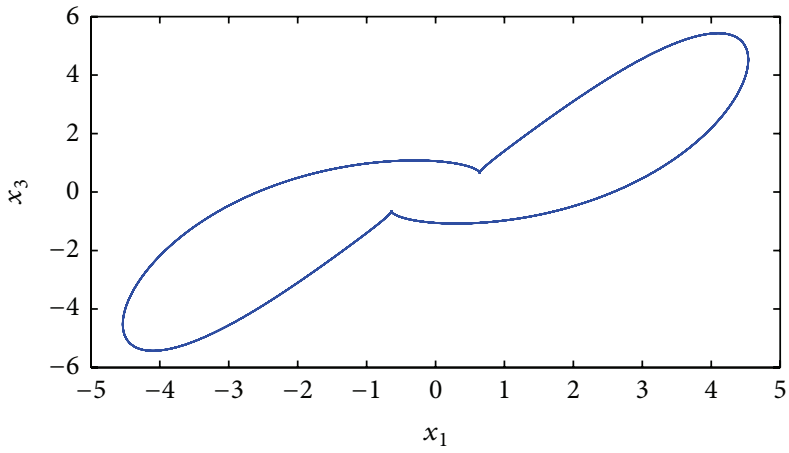

(a)

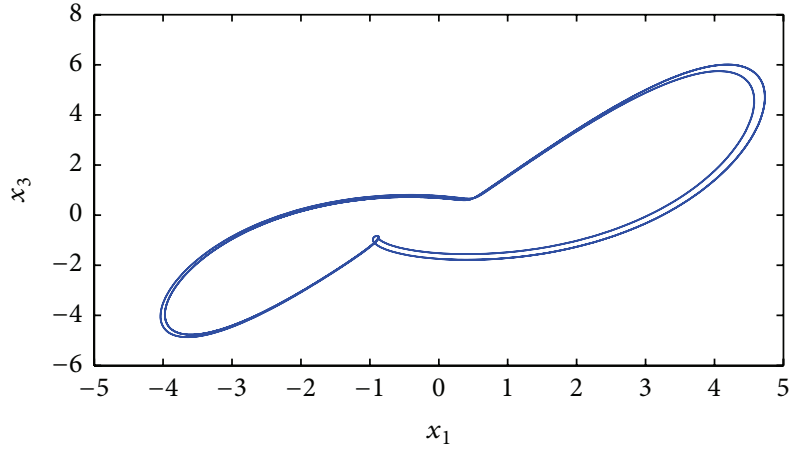

(b)

FIGURE 3: The phase portraits of the system for different values of the parameter $a$ : (a) the phase trajectory for $a=7$ and (b) the phase trajectory for $a=5.8$.

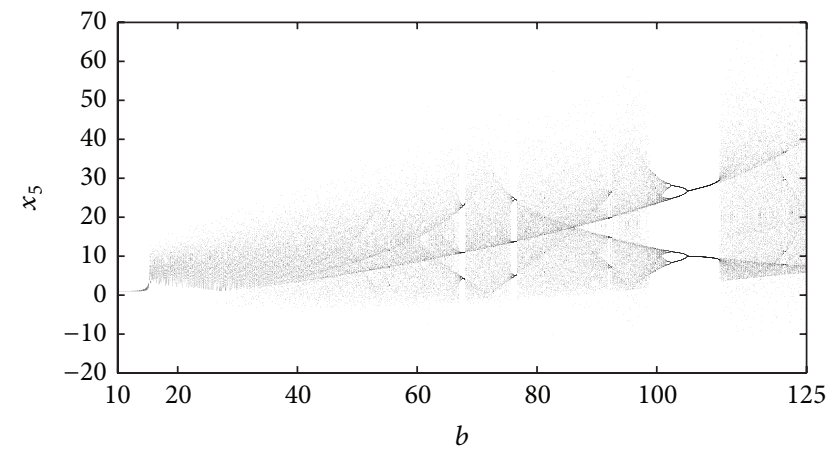

FIGURE 4: The bifurcation diagram of the system (14) with parameter $b \in[10,125]$.

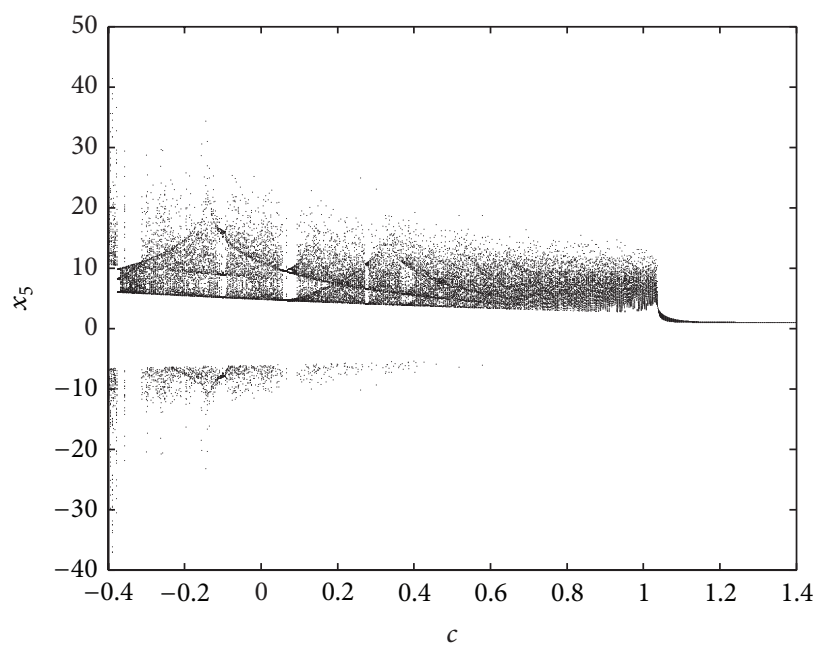

FIGURE 5: The bifurcation diagram of the system (18) with parameter $c \in[-0.4,1.4]$.

Multiplying the above equation left by $\xi^{H} \mathbf{P}$, we obtain that

$$
\xi^{H} \mathbf{P}[A(y, z)+B(y, z)] \xi=\xi^{H} \mathbf{P}(\lambda \xi) .
$$

Then, by a similar argument, we also can get that

$$
\left\{\xi^{H}[A(y, z)+B(y, z)]^{H}\right\} \mathbf{P} \xi=\left(\bar{\lambda} \xi^{H}\right) \mathbf{P} \xi .
$$

From (26) and (27), we derive

$$
\begin{aligned}
\lambda+\bar{\lambda}=\left\{\xi^{H}\{\mathbf{P}[A(y, z)+B(y, z)]\right. \\
\left.\left.+[A(y, z)+B(y, z)]^{H} \mathbf{P}\right\} \xi\right\}\left(\xi^{H} \mathbf{P} \xi\right)^{-1} .
\end{aligned}
$$

Since $\mathbf{P}[A(y, z)+B(y, z)]+[A(y, z)+B(y, z)]^{H} \mathbf{P}=-\mathbf{Q}$ and $\mathbf{P}$ and $\mathbf{Q}$ are real symmetric positive definite matrices, then

$$
\beta^{H} \mathbf{Q} \beta>0, \quad \beta^{H} \mathbf{P} \beta>0, \quad \lambda+\bar{\lambda}=-\frac{\beta^{H} \mathbf{Q} \beta}{\beta^{H} \mathbf{P} \beta}<0 .
$$

From the above formula, we obtain

$$
|\arg \lambda| \geq \frac{\pi}{2}>\frac{\pi}{2} q, \quad(0<q<1) .
$$

According to the stability theory of fractional-order systems, the equilibrium point of error dynamical system (23) is asymptotically stable. Therefore, the synchronization condition $\lim _{t \rightarrow \infty}\|e\|=\lim _{t \rightarrow \infty}\|z-y\|=0$ is satisfied, which implies that the synchronization between drive and response systems is realized. The proof is completed.

4.2. Numerical Simulation. The system (14) is taken as the drive system, and the corresponding response system with controller is

$$
\left[\begin{array}{c}
D^{\beta} z_{1} \\
D^{\beta} z_{2} \\
D^{\beta} z_{3}
\end{array}\right]=\left[\begin{array}{c}
a\left(z_{2}-z_{1}\right) \\
z_{1} z_{3}-z_{1} \\
b-\frac{1}{2}\left(\bar{z}_{1} z_{2}+z_{1} \bar{z}_{2}\right)-c z_{3}
\end{array}\right]+u(y, z),
$$




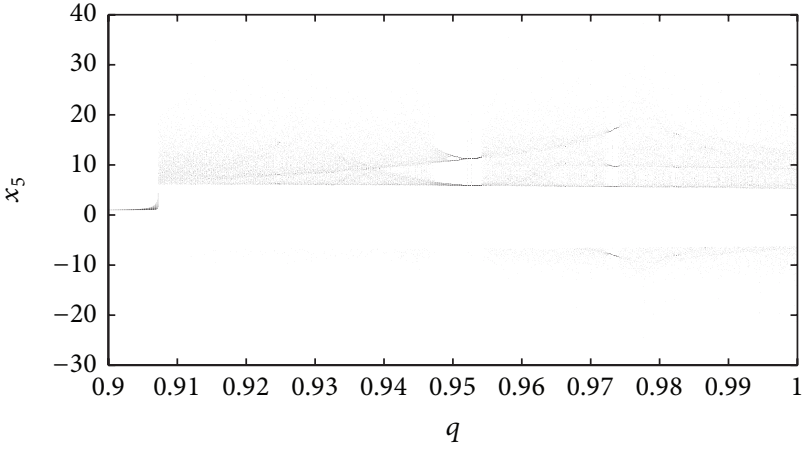

(a)

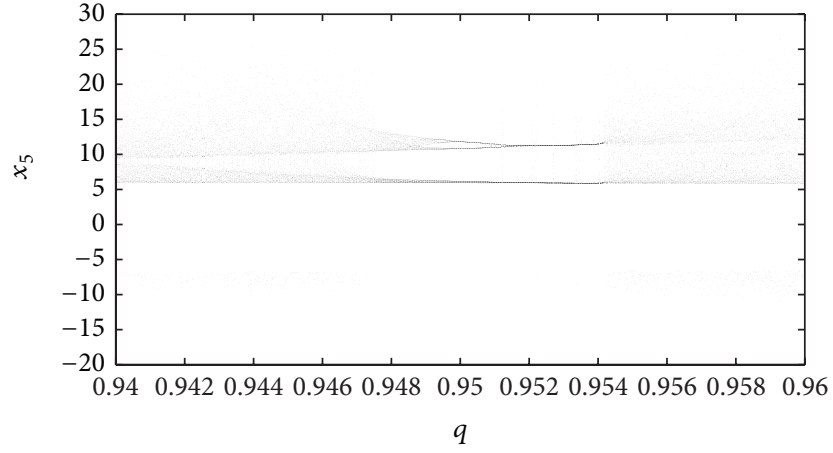

(b)

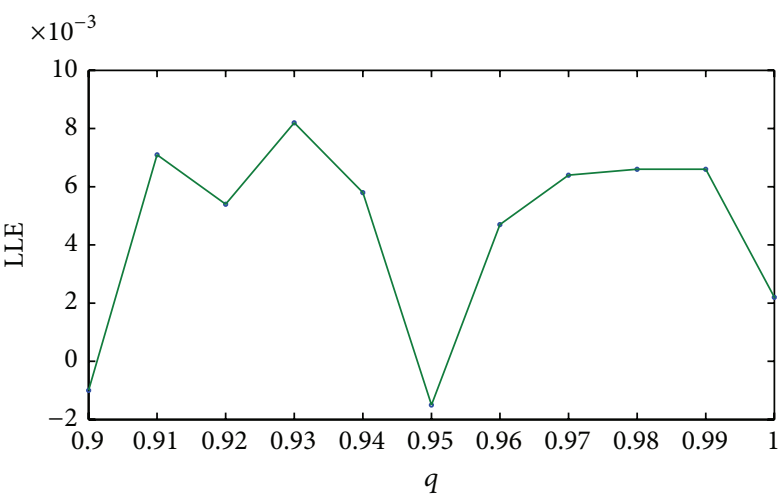

(c)

FIgURE 6: The bifurcation diagram of the system (14) with derivative order $q$. (a) $q \in[0.9,1]$; (b) local zoom of (a); (c) the corresponding LLE diagram.

where $z_{1}=x_{1}^{\prime}+i x_{2}^{\prime}$ and $z_{2}=x_{3}^{\prime}+i x_{4}^{\prime}$ are complex variables and $z_{3}=x_{5}^{\prime}$ is real variable, and $i=\sqrt{-1}$. The system (31) can be expressed as

$$
\left[\begin{array}{c}
D^{\beta} x_{1}^{\prime} \\
D^{\beta} x_{2}^{\prime} \\
D^{\beta} x_{3}^{\prime} \\
D^{\beta} x_{4}^{\prime} \\
D^{\beta} x_{5}^{\prime}
\end{array}\right]=\left[\begin{array}{c}
a\left(x_{3}^{\prime}-x_{1}^{\prime}\right) \\
a\left(x_{4}^{\prime}-x_{2}^{\prime}\right) \\
x_{1}^{\prime} x_{5}^{\prime}-x_{1}^{\prime} \\
x_{2}^{\prime} x_{5}^{\prime}-x_{2}^{\prime} \\
b-\left(x_{1}^{\prime} x_{3}^{\prime}+x_{2}^{\prime} x_{4}^{\prime}\right)-c x_{5}^{\prime}
\end{array}\right]+u(y, z) .
$$

The synchronization error vector is taken as

$$
e=z-y=e^{\text {real }}+i e^{\text {image }}
$$

where $e^{\text {real }}=\left(e_{1}, e_{3}, e_{5}\right)^{T}$ and $e^{\text {image }}=\left(e_{2}, e_{4}\right)^{T}$.

By computation, we can get $A\left(x_{j}, x_{j}^{\prime}\right)$ as follows:

$$
A\left(x_{j}, x_{j}^{\prime}\right)=\left[\begin{array}{ccccc}
-a & 0 & a & 0 & 0 \\
0 & -a & 0 & a & 0 \\
x_{5}^{\prime} & 0 & -1 & 0 & x_{1} \\
0 & x_{5}^{\prime} & 0 & -1 & x_{2} \\
-x_{3}^{\prime} & -x_{4}^{\prime} & -x_{1} & -x_{2} & -c
\end{array}\right]
$$

By using the theorem, the matrix $B\left(x_{j}, x_{j}^{\prime}\right)$ is designed as

$$
B\left(x_{j}, x_{j}^{\prime}\right)=\left[\begin{array}{ccccc}
0 & 0 & 0 & 0 & x_{3}^{\prime} \\
0 & 0 & 0 & 0 & x_{4}^{\prime} \\
-x_{5}^{\prime}-a & 0 & 0 & 0 & 0 \\
0 & -x_{5}^{\prime}-a & 0 & 0 & 0 \\
0 & 0 & 0 & 0 & 0
\end{array}\right] .
$$

Therefore, the error dynamical system is

$$
\left[\begin{array}{l}
D^{\beta} e_{1} \\
D^{\beta} e_{2} \\
D^{\beta} e_{3} \\
D^{\beta} e_{4} \\
D^{\beta} e_{5}
\end{array}\right]=\left[\begin{array}{ccccc}
-a & 0 & a & 0 & x_{3}^{\prime} \\
0 & -a & 0 & a & x_{4}^{\prime} \\
-a & 0 & -1 & 0 & x_{1} \\
0 & -a & 0 & -1 & x_{2} \\
-x_{3}^{\prime} & -x_{4}^{\prime} & -x_{1} & -x_{2} & -c
\end{array}\right]\left[\begin{array}{l}
e_{1} \\
e_{2} \\
e_{3} \\
e_{4} \\
e_{5}
\end{array}\right] .
$$

A real symmetric positive definite matrix is chosen as $\mathbf{P}=$ $\operatorname{diag}(1,1,1,1,1)$; then,

$$
\begin{gathered}
P\left[A\left(x_{j}, x_{j}^{\prime}\right)+B\left(x_{j}, x_{j}^{\prime}\right)\right]+\left[A\left(x_{j}, x_{j}^{\prime}\right)+B\left(x_{j}, x_{j}^{\prime}\right)\right]^{H} \mathbf{P} \\
\quad=\operatorname{diag}(-2 a,-2 a,-2,-2,-2 c) .
\end{gathered}
$$




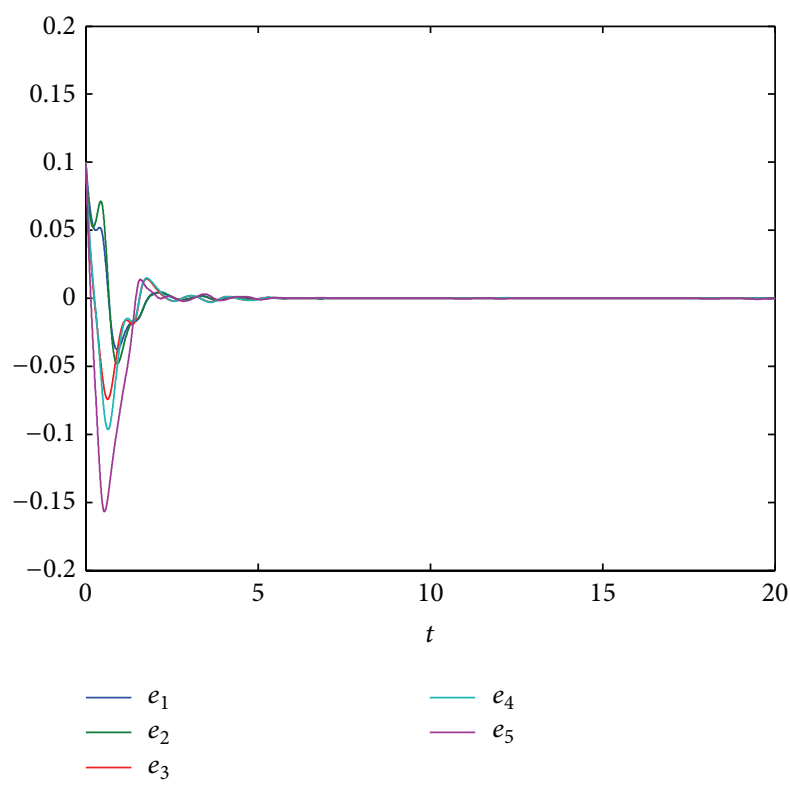

FIGURE 7: Curves of the synchronization errors of the systems (14) and (32).

Therefore, another real symmetric positive definite matrix is taken as $\mathbf{Q}=\operatorname{diag}(2 a, 2 a, 2,2,2 c)$, and expression (37) can be written as

$$
\begin{aligned}
\mathbf{P}\left[A\left(x_{j}, x_{j}^{\prime}\right)+B\left(x_{j}, x_{j}^{\prime}\right)\right] \\
+\left[A\left(x_{j}, x_{j}^{\prime}\right)+B\left(x_{j}, x_{j}^{\prime}\right)\right]^{H} P=-\mathbf{Q} .
\end{aligned}
$$

According to the theorem, the synchronization of drive system (14) and response system (32) is achieved.

The values of the parameters of the drive and response systems are taken as $a=5, b=16, c=1$. The orders of derivative for the two systems are $q=\beta=0.99$. The initial conditions of the two systems are $(0.1,0.1,0.1,0.1,0.1)$ and $(1,2,3,4,5)$, respectively. The results of simulation are depicted in Figure 7, from which it is clear that the error variables $e_{1}, e_{2}, e_{3}, e_{4}$, and $e_{5}$ converge to zero as $t \rightarrow 20 \mathrm{~s}$, which implies the synchronization between the drive and response systems is achieved under the controllers.

\section{Conclusions}

In this paper, the dynamics and synchronization for a fractional-order system with complex variables have been investigated. The dynamics include symmetry, equilibrium points, chaos, and bifurcations with variation of both system parameters and derivative orders. Besides, based on the stability theory of fractional-order systems, the scheme of synchronization for the fractional-order complex system is presented, and numerical simulations are carried out to demonstrate the effectiveness and feasibility of the proposed scheme.

\section{Conflict of Interests}

The authors declare that there is no conflict of interests regarding the publication of this paper.

\section{Acknowledgment}

The authors are very grateful to the anonymous reviewers for the insightful comments leading to the improvement of the paper.

\section{References}

[1] C. P. Li, W. H. Deng, and D. Xu, "Chaos synchronization of the Chua system with a fractional order," Physica A: Statistical Mechanics and Its Applications, vol. 360, no. 2, pp. 171-185, 2006.

[2] P. Arena, R. Caponetto, L. Fortuna, and D. Porto, "Chaos in a fractional order Duffing system," in Proceedings of the European Conference on Circuit Theory and Design (ECCTD '97), pp. 1259$1262,1997$.

[3] J. G. Lu and G. Chen, "A note on the fractional-order Chen system," Chaos, Solitons and Fractals, vol. 27, no. 3, pp. 685-688, 2006.

[4] L. J. Sheu, W. C. Chen, Y. C. Chen, and W. T. Weng, "A twochannel secure communication using fractional chaotic systems," World Academy of Science, Engineering and Technology, vol. 65, pp. 1057-1061, 2010.

[5] A. Kiani-B, K. Fallahi, N. Pariz, and H. Leung, "A chaotic secure communication scheme using fractional chaotic systems based on an extended fractional Kalman filter," Communications in Nonlinear Science and Numerical Simulation, vol. 14, no. 3, pp. 863-879, 2009.

[6] L. M. Pecora and T. L. Carroll, "Synchronization in chaotic systems," Physical Review Letters, vol. 64, no. 8, pp. 821-824, 1990.

[7] G. M. Mahmoud, T. Bountis, G. M. AbdEl-Latif, and E. E. Mahmoud, "Chaos synchronization of two different chaotic complex Chen and Lü systems," Nonlinear Dynamics, vol. 55, no. 1-2, pp. 43-53, 2009.

[8] G. M. Mahmoud and E. E. Mahmoud, "Complete synchronization of chaotic complex nonlinear systems with uncertain parameters," Nonlinear Dynamics, vol. 62, no. 4, pp. 875-882, 2010.

[9] G. M. Mahmoud and E. E. Mahmoud, "Synchronization and control of hyperchaotic complex Lorenz system," Mathematics and Computers in Simulation, vol. 80, no. 12, pp. 2286-2296, 2010.

[10] G. Wu and D. Baleanu, "Discrete fractional logistic map and its chaos," Nonlinear Dynamics, vol. 75, no. 1-2, pp. 283-287, 2014.

[11] G. C. Wu and D. Baleanu, "Discrete chaos in fractional delayed logistic maps," Nonlinear Dynamics, 2014.

[12] G. C. Wu and D. Baleanu, "Chaos synchronization of the discrete fractional logistic map," Signal Processing, vol. 102, pp. 9699, 2014.

[13] G. C. Wu, D. Baleanu, and S. D. Zeng, "Discrete chaos in fractional sine and standard maps," Physics Letters A, vol. 378, no. 5-6, pp. 484-487, 2014.

[14] I. Podlubny, Fractional Differential Equations, Academic Press, San Diego, Calif, USA, 1999.

[15] X. J. Liu, L. Hong, and L. X. Yang, "Fractional-order complex T system: bifurcations, chaos control, and synchronization," Nonlinear Dynamics, vol. 75, no. 3, pp. 589-602, 2014. 
[16] E. Hairer, S. P. Nørsett, and G. Wanner, Solving Ordinary Differential Equations I: Nonstiff Problems, vol. 8 of Springer Series in Computational Mathematics, Springer, Berlin, Germany, 2nd edition, 1993.

[17] E. Hairer and G. Wanner, Solving Ordinary Differential Equations II: Stiff and Differential-Algebraic Problems, Springer, Berlin, Germany, 1991.

[18] K. Diethelm, N. J. Ford, and A. D. Freed, "A predictor-corrector approach for the numerical solution of fractional differential equations," Nonlinear Dynamics, vol. 29, no. 1-4, pp. 3-22, 2002.

[19] A. Charef, H. H. Sun, Y. Tsao, and B. Onaral, "Fractal system as represented by singularity function," IEEE Transactions on Automatic Control, vol. 37, no. 9, pp. 1465-1470, 1992.

[20] X. Li, Y. Chu, J. Zhang, and Y. Chang, "Nonlinear dynamics and circuit implementation for a new Lorenz-like attractor," Chaos, Solitons and Fractals, vol. 41, no. 5, pp. 2360-2370, 2009.

[21] A. K. Golmankhaneh, R. Arefi, and D. Baleanu, "The proposed modified Liu system with fractional order," Advances in Mathematical Physics, vol. 2013, Article ID 186037, 6 pages, 2013.

[22] A. K. Golmankhaneh, A. Roohiyeh, and D. Baleanu, "Synchronization in a nonidentical fractional order of a proposed modified system," Journal of Vibration and Control, 2013. 


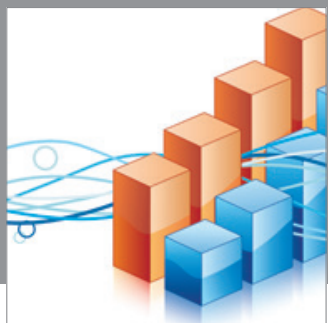

Advances in

Operations Research

mansans

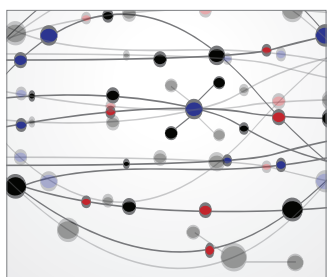

The Scientific World Journal
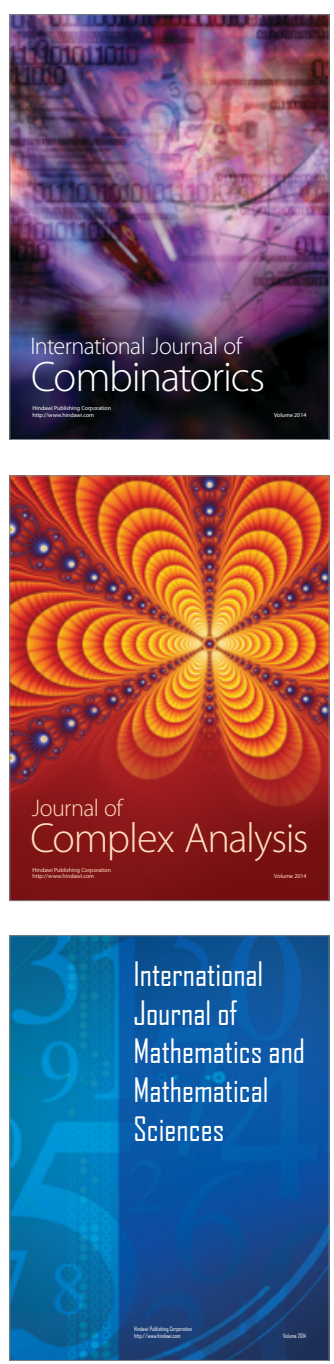
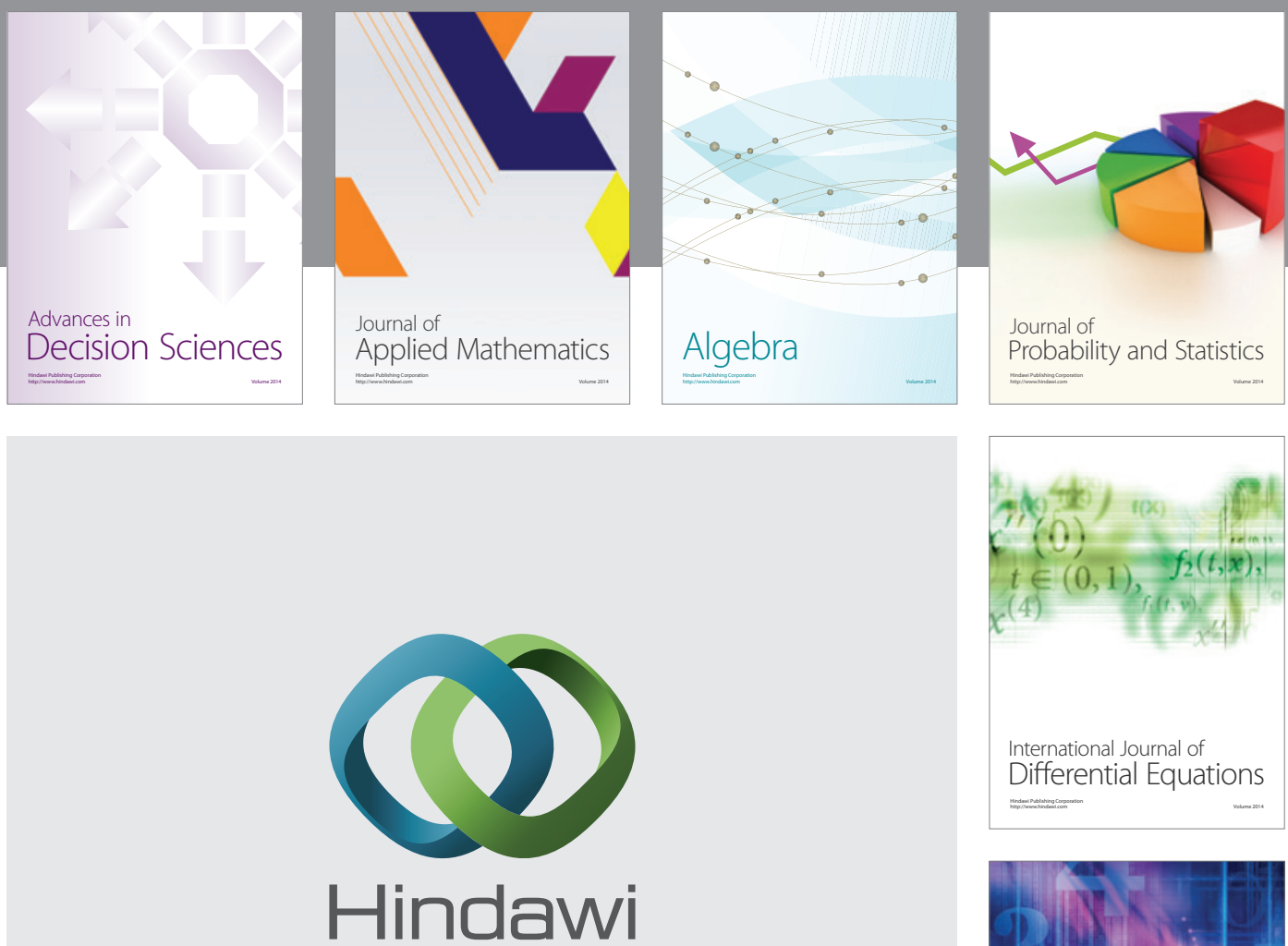

Submit your manuscripts at http://www.hindawi.com
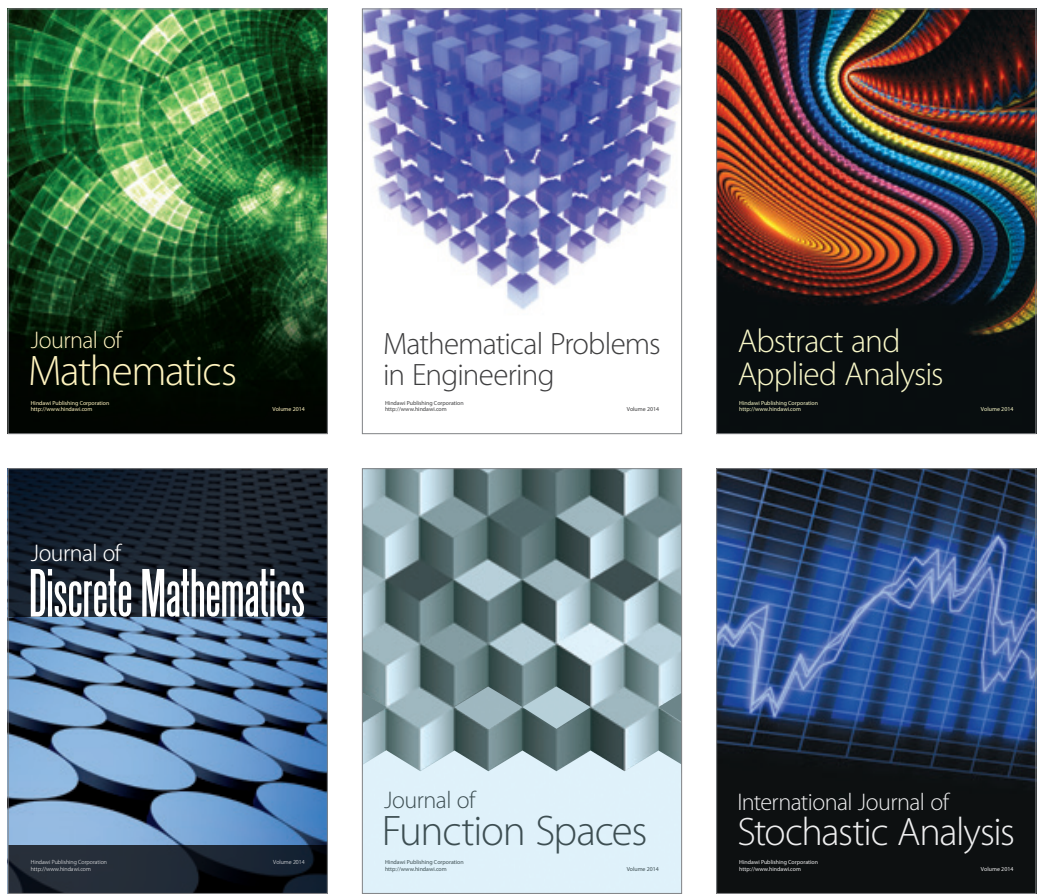

Journal of

Function Spaces

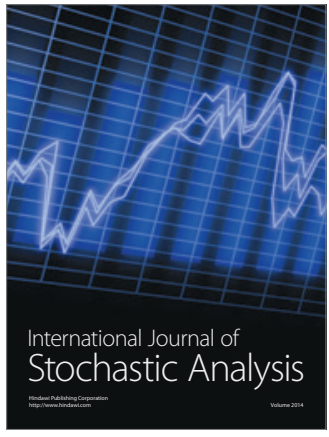

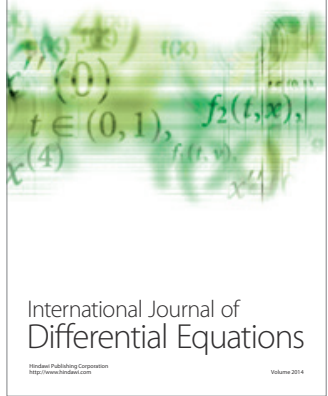
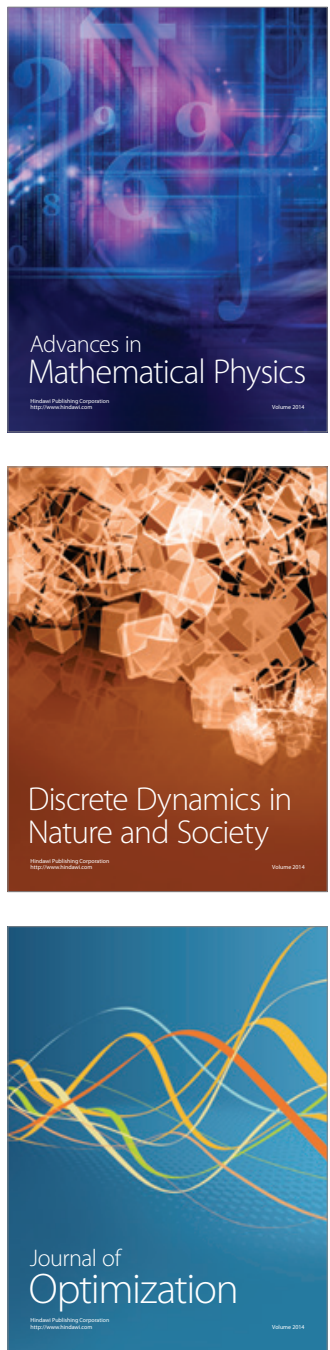